\title{
Avaliação clínica e radiológica dos pacientes submetidos a osteossíntese de rádio distal com placas bloqueadas - estudo retrospectivo*
}

\section{Clinical and Radiological Evaluation of Patients Undergoing Distal Radio Osteosynthesis with Locking Plate - Retrospective Study}

\author{
Jurandyr de Abreu Câmara Filho ${ }^{1}$ Saulo Fontes Almeida ${ }^{1}$
} ${ }^{1}$ Serviço Clínico de Ortopedia e Traumatologia, Hospital Naval
Marcílio Dias, Rio de Janeiro, RJ, Brasil

Rev Bras Ortop 2019;54:303-308.
Address for correspondence Jurandyr de Abreu Câmara Filho, PhD, Rua Petrolândia 480 Vista Alegre, Rio de Janeiro, RJ, CEP: 21230-720, Brasil (e-mail: jurandyrabreu@id.uff.br).

\section{Resumo}

Palavras-chave

- fraturas ósseas

- fraturas do rádio

- fixação interna de fraturas

- estudos retrospectivos

\section{Abstract}

Objetivo O objetivo do presente trabalho foi realizar um estudo retrospectivo, com base no arquivo médico dos prontuários e das radiografias dos pacientes, avaliando seus dados socioeconômicos, assim como clinicamente e radiograficamente, os pacientes submetidos a osteossíntese de rádio distal com placas bloqueadas.

Métodos Avaliou-se clinicamente e radiologicamente o pós-operatório, no Serviço da clínica de Ortopedia e Traumatologia do nosso hospital, entre os anos de 2016 e 2017. Resultados $\mathrm{Na}$ avaliação radiográfica, foram encontrados $22(75,86 \%)$ resultados excelentes, $6(20,69 \%)$ bons e $1(3,45 \%)$ regular. Na avaliação clínica, o desvio ulnar obteve média de 28,40 , desvio padrão (DP) de 3,0 e coeficiente de variação de 10,56\%. O desvio radial obteve média de 22,93 , DP de 2,2 e coeficiente de variação de $9,59 \%$. A amplitude de movimento em flexão obteve média de 59,43 , DP de 9,86 e coeficiente de variação de $16,59 \%$. Já a extensão obteve média de 53,83, DP de 5,09 e coeficiente de variação de $9,46 \%$.

Conclusão Concluímos que há correlação estatística entre os dados clínicos e radiográficos, e que a placa bloqueada é um método de tratamento com alto índice de sucesso no procedimento cirúrgico das fraturas de rádio distal.

Objective The objective of the present work was to perform a retrospective study, based on the medical files and radiographs of the patients, evaluating clinically and radiographically, as well as the socioeconomic data of the patients submitted to distal radio osteosynthesis with locking plates.

Methods The postoperative period was evaluated clinically and radiologically in the Orthopedics and Traumatology Clinic Service of our hospital between 2016 and 2017. Results In the radiographic evaluation 22 (75.86\%) excellent results, $6(20.69 \%)$ good results, and 1 (3.45\%) regular result were found. In the clinical evaluation, the ulnar

Trabalho realizado no Hospital Naval Marcílio Dias, Rio de Janeiro, RJ, Brasil.

received

December 27, 2017

accepted

May 28, 2018
DOI https://doi.org/

10.1055/s-0039-1691759. ISSN 0102-3616.
Copyright $\odot 2019$ by Sociedade Brasileira License terms de Ortopedia e Traumatologia. Published by Thieme Revnter Publicações Ltda, Rio de Janeiro, Brazil 
Keywords

- fractures, bone

- radius fractures

- internal fracture fixation

- retrospective studies deviation obtained a mean of 28.40, a standard deviation (SD) of 3.0 and a coefficient of variation of $10.56 \%$. The radial deviations averaged 22.93 , with a SD of 2.2 and a coefficient of variation of $9.59 \%$. The range of motion in flexion obtained a mean of 59.43 , a SD of 9.86 and a coefficient of variation of $16.59 \%$. The extension obtained a mean of 53.83, a SD of 5.09 and a coefficient of variation of $9.46 \%$.

Conclusion We have concluded that there is statistical correlation between clinical and radiographic data, and that the locking plate is a treatment method with a high success rate in the surgical procedure of distal radius fractures.

\section{Introdução}

As fraturas do rádio distal são lesões frequentes, respondendo por entre $10 \mathrm{a} 12 \%$ das fraturas do esqueleto humano. A maioria ocorre após trauma de baixa energia, como queda da própria altura, e está relacionada à perda de densidade óssea (osteoporose). ${ }^{1}$

Nos pacientes jovens, por outro lado, as fraturas do rádio estão associadas a traumas de grande energia, como os que ocorrem nos acidentes de trânsito, esportivos e quedas de altura.

Na literatura, não há um único método de tratamento que seja eficaz para todos os tipos de fraturas do rádio distal. ${ }^{2,3}$

Dentre as formas de tratamento descritas, estão a imobilização gessada, o uso de fios de Kirschner percutâneos intrafocais, extrafocais ou intramedulares, placas dorsais e volares, e fixação externa. ${ }^{4,5}$

Os recentes avanços nos implantes e nas técnicas, como as placas bloqueadas, têm mudado o tratamento ortopédico dessas fraturas. Os sistemas de placa e parafuso bloqueados agem como uma unidade para a fixação da fratura, ao contrário das placas convencionais, que necessitam de compressão entre o implante e o osso para a estabilização. Nos pacientes idosos, que possuem uma menor densidade óssea, os sistemas de placa e parafuso bloqueados fornecem maior rigidez e melhor estabilização do que as placas convencionais. Além disso, o mecanismo parafuso-placa bloqueada funciona como um substituto da cortical óssea, permitindo a utilização de parafusos monocorticais. ${ }^{6}$

A fixação interna estável possui vantagens, incluindo a mobilização precoce do punho e dos dedos, facilitando a reabilitação pós-operatória e diminuindo a rigidez por tempo de imobilização. ${ }^{7}$

Estudos biomecânicos demonstram maior estabilidade nas fraturas fixadas com placas bloqueadas em relação às placas não bloqueadas, dorsais ou volares. As placas volares possuem a vantagem de provocar menor incidência de complicações relacionadas aos tendões extensores quando comparadas às dorsais. ${ }^{8}$

O objetivo do tratamento cirúrgico para as fraturas instáveis do rádio distal é obter redução anatômica e permitir uma melhor recuperação funcional do paciente ${ }^{9,10}$ No entanto, estudos mostram não haver correlação entre o restabelecimento da inclinação volar, do comprimento radial e da inclinação radial com melhores resultados funcionais subjetivos de pacientes tratados com imobilização gessada ou com fios de
Kirschner. ${ }^{9}$ A relação dessas medidas radiográficas com a recuperação da amplitude de movimento é controversa.

o comprimento radial e a angulação volar são as mais citadas como responsáveis por repercussões no movimento do punho dos pacientes tratados por fraturas do rádio distal. ${ }^{10}$

O objetivo do presente trabalho foi realizar um estudo retrospectivo, com base no arquivo médico dos prontuários e das radiografias dos pacientes, avaliando seus dados socioeconômicos, assim como clinicamente e radiograficamente, os pacientes submetidos a osteossíntese de rádio distal com placas bloqueadas.

\section{Material e Métodos}

Realizamos um estudo retrospectivo, com base no arquivo médico dos prontuários e das radiografias de 30 pacientes admitidos no Serviço da clínica de Ortopedia e Traumatologia do Hospital Naval Marcílio Dias, submetidos a procedimento cirúrgico para o tratamento das fraturas da extremidade distal do rádio, com placas com desenho de rádio distal, de titânio, dupla coluna distal e bloqueadas com ângulo variável, operados pelos cirurgiões Almeida S. F. e Câmara Filho J. A., entre 2016 e 2017. Foram excluídos pacientes com esqueleto imaturo. Projeto aprovado pelo CEP com o número 2.447.319.

Os seguintes dados sociodemográficos dos pacientes foram obtidos e registrados: idade, gênero, data da cirurgia, lado e mão dominante, dentre outros ( - Tabela 1 ).

Foram utilizadas classificações pré-operatoriamente através de radiografias nas incidências anteroposterior e lateral do punho, de acordo com a classificação universal, proposta por Cooney e Berger e com a classificação AO.

Os critérios de Lafontaine de instabilidade foram utilizados como parâmetros de estudos para a indicação do procedimento cirúrgico em questão, a saber: desvio dorsal $>20^{\circ}$, cominuição dorsal, encurtamento do rádio $>9$ $\mathrm{mm}$, envolvimento articular radiocárpico e radioulnar distal, fraturas associadas da ulna, afastamento entre os fragmentos intra-articulares $>2 \mathrm{~mm}$ e idade $>60$ anos.

Clinicamente, foram observadas as amplitudes de movimentos do punho através da flexão, da extensão, do desvio radial e ulnar entre 6 a 8 meses de pós-operatório. As medições dos arcos de movimento foram efetuadas por pelo menos dois dos autores, e os valores da média entre eles foram documentados com a utilização de um goniômetro. 
Tabela 1 Avaliação clínica

\begin{tabular}{|c|c|c|c|c|c|c|c|c|}
\hline Paciente & Idade & Gênero & $\begin{array}{l}\text { Membro superior } \\
\text { acometido }\end{array}$ & $\begin{array}{l}\text { Desvio } \\
\text { ulnar }\end{array}$ & $\begin{array}{l}\text { Desvio } \\
\text { adial }\end{array}$ & Flexão & Extensão & $\begin{array}{l}\text { Avaliação } \\
\text { radiológica }\end{array}$ \\
\hline 1 & 91 & $\mathrm{M}$ & $\mathrm{E}$ & $26^{\circ}$ & $21^{\circ}$ & $55^{\circ}$ & $50^{\circ}$ & Bom \\
\hline 2 & 66 & $\mathrm{M}$ & $E$ & $29^{\circ}$ & $20^{\circ}$ & $58^{\circ}$ & $49^{\circ}$ & Bom \\
\hline 3 & 49 & $\mathrm{~F}$ & $\mathrm{D}$ & $28^{\circ}$ & $25^{\circ}$ & $62^{\circ}$ & $58^{\circ}$ & Excelente \\
\hline 4 & 58 & $\mathrm{~F}$ & $\mathrm{E}$ & $26^{\circ}$ & $25^{\circ}$ & $40^{\circ}$ & $50^{\circ}$ & Excelente \\
\hline 5 & 63 & M & D & $29^{\circ}$ & $26^{\circ}$ & $45^{\circ}$ & $52^{\circ}$ & Bom \\
\hline 6 & 54 & $F$ & $\mathrm{D}$ & $28^{\circ}$ & $25^{\circ}$ & $59^{\circ}$ & $58^{\circ}$ & Excelente \\
\hline 7 & 31 & $\mathrm{~F}$ & $E$ & $30^{\circ}$ & $25^{\circ}$ & $62^{\circ}$ & $59^{\circ}$ & Excelente \\
\hline 8 & 43 & $\mathrm{M}$ & $E$ & $30^{\circ}$ & $25^{\circ}$ & $80^{\circ}$ & $50^{\circ}$ & Bom \\
\hline 9 & 76 & $\mathrm{~F}$ & D & $29^{\circ}$ & $24^{\circ}$ & $56^{\circ}$ & $52^{\circ}$ & Excelente \\
\hline 10 & 40 & $\mathrm{~F}$ & $\mathrm{D}$ & $31^{\circ}$ & $23^{\circ}$ & $63^{\circ}$ & $60^{\circ}$ & Excelente \\
\hline 11 & 67 & $\mathrm{~F}$ & $E$ & $25^{\circ}$ & $23^{\circ}$ & $55^{\circ}$ & $50^{\circ}$ & Excelente \\
\hline 12 & 63 & $\mathrm{~F}$ & $\mathrm{D}$ & $34^{\circ}$ & $25^{\circ}$ & $48^{\circ}$ & $56^{\circ}$ & Excelente \\
\hline 13 & 79 & $\mathrm{~F}$ & E & $30^{\circ}$ & $25^{\circ}$ & $80^{\circ}$ & $50^{\circ}$ & Bom \\
\hline 14 & 50 & $\mathrm{M}$ & $\mathrm{E}$ & $30^{\circ}$ & $25^{\circ}$ & $70^{\circ}$ & $63^{\circ}$ & Excelente \\
\hline 15 & 70 & M & $E$ & $26^{\circ}$ & $24^{\circ}$ & $45^{\circ}$ & $50^{\circ}$ & Excelente \\
\hline 16 & 52 & $\mathrm{M}$ & D & $31^{\circ}$ & $22^{\circ}$ & $55^{\circ}$ & $53^{\circ}$ & Bom \\
\hline 17 & 83 & $\mathrm{~F}$ & $E$ & $21^{\circ}$ & $23^{\circ}$ & $55^{\circ}$ & $52^{\circ}$ & Regular \\
\hline 18 & 68 & $\mathrm{~F}$ & $\mathrm{E}$ & $30^{\circ}$ & $23^{\circ}$ & $80^{\circ}$ & $68^{\circ}$ & Excelente \\
\hline 19 & 83 & $\mathrm{~F}$ & $E$ & $30^{\circ}$ & $20^{\circ}$ & $60^{\circ}$ & $55^{\circ}$ & Excelente \\
\hline 20 & 84 & $\mathrm{~F}$ & $E$ & $32^{\circ}$ & $22^{\circ}$ & $55^{\circ}$ & $48^{\circ}$ & Excelente \\
\hline 21 & 32 & M & $\mathrm{D}$ & $30^{\circ}$ & $23^{\circ}$ & $60^{\circ}$ & $56^{\circ}$ & Excelente \\
\hline 22 & 49 & $M$ & $E$ & $30^{\circ}$ & $21^{\circ}$ & $72^{\circ}$ & $55^{\circ}$ & Excelente \\
\hline 23 & 68 & $\mathrm{~F}$ & $E$ & $25^{\circ}$ & $20^{\circ}$ & $68^{\circ}$ & $45^{\circ}$ & Excelente \\
\hline 24 & 80 & $\mathrm{~F}$ & $\mathrm{E}$ & $26^{\circ}$ & $19^{\circ}$ & $63^{\circ}$ & $48^{\circ}$ & Excelente \\
\hline 25 & 83 & $\mathrm{~F}$ & $\mathrm{D}$ & $27^{\circ}$ & $18^{\circ}$ & $60^{\circ}$ & $49^{\circ}$ & Excelente \\
\hline 26 & 58 & $\mathrm{~F}$ & D & $30^{\circ}$ & $25^{\circ}$ & $55^{\circ}$ & $53^{\circ}$ & Excelente \\
\hline 27 & 70 & $\mathrm{~F}$ & $E$ & $30^{\circ}$ & $24^{\circ}$ & $55^{\circ}$ & $52^{\circ}$ & Excelente \\
\hline 28 & 20 & $\mathrm{M}$ & $\mathrm{E}$ & $20^{\circ}$ & $25^{\circ}$ & $58^{\circ}$ & $60^{\circ}$ & Excelente \\
\hline 29 & 63 & $\mathrm{~F}$ & $E$ & $30^{\circ}$ & $22^{\circ}$ & $50^{\circ}$ & $58^{\circ}$ & Excelente \\
\hline 30 & 58 & $\mathrm{~F}$ & D & $29^{\circ}$ & $20^{\circ}$ & $59^{\circ}$ & $56^{\circ}$ & \\
\hline
\end{tabular}

Abreviações: D, direito; E, esquerdo; F, feminino; M, masculino.

Utilizamos radiografias nas incidências anteroposterior e lateral de ambos os punhos, para fins comparativos de controle da redução cirúrgica. O controle radiográfico foi avaliado de acordo com a classificação anatomorradiológica para as fraturas da extremidade distal do rádio de Lidström em excelente, bom, regular e ruim (-Tabela 2 ).

Os resultados clínicos e radiográficos foram analisados e comparados.

\section{Resultados}

Os resultados dos 30 pacientes encontram-se na - Tabela 1, sendo que $030^{\circ}$ paciente não tem em seu prontuário a avaliação radiológica.
Não foram observadas infecções, e as fraturas estavam consolidadas em todos os casos.

Analisando os resultados, verificou-se que as idades dos pacientes variaram de 20 a 91 anos, com média de 56 anos. Em relação ao gênero, 66,6\% dos pacientes eram do sexo feminino, e 33,3\% do masculino. Em relação ao lado acometido, $63,3 \%$ das fraturas ocorreram no lado esquerdo, e $36,3 \%$ no direito. Na avaliação radiográfica, foram encontrados 22 (75,86\%) resultados excelentes, $6(20,69 \%)$ bons, e 1 (3,45\%) regular, conforme demonstrado na - Fig. 1.

Na avaliação clínica, o desvio ulnar obteve média de 28,40, desvio padrão (DP) de 3,0 e coeficiente de variação de 10,56\%. O desvio radial obteve média de 22,93, DP de 2,2 e coeficiente de variação de $9,59 \%$. A amplitude de movimento em flexão 
Tabela 2 Classificação anatomorradiólogica para as fraturas da extremidade distal do rádio segundo Lidstrôm

\begin{tabular}{|l|}
\hline Excelente \\
\hline Deformidade insignificante: \\
\hline - Angulação dorsal $>0^{\circ}$ (neutro) \\
\hline - Encurtamento radial $<3 \mathrm{~mm}$ \\
\hline - Perda da inclinação radial não $>4^{\circ}$ \\
\hline Bom \\
\hline Deformidade pequena: \\
\hline - Angulação dorsal entre $1^{\circ}$ e $10^{\circ}$ \\
\hline - Encurtamento radial entre $3 \mathrm{~mm}$ e $6 \mathrm{~mm}$ \\
\hline - Perda da inclinação radial entre $5^{\circ}$ e $9^{\circ}$ \\
\hline Regular \\
\hline Deformidade moderada: \\
\hline - Angulação dorsal entre $11^{\circ}$ e $14^{\circ}$ \\
\hline - Encurtamento radial entre $7 \mathrm{~mm}$ e $11 \mathrm{~mm}$ \\
\hline - Perda da inclinação radial entre $10^{\circ}$ e $14^{\circ}$ \\
\hline Ruim \\
\hline Deformidade severa: \\
\hline - Angulação dorsal $>15^{\circ}$ \\
\hline - Encurtamento radial $>11 \mathrm{~mm}$ \\
\hline - Perda da inclinação radial $>15^{\circ}$ \\
\hline
\end{tabular}

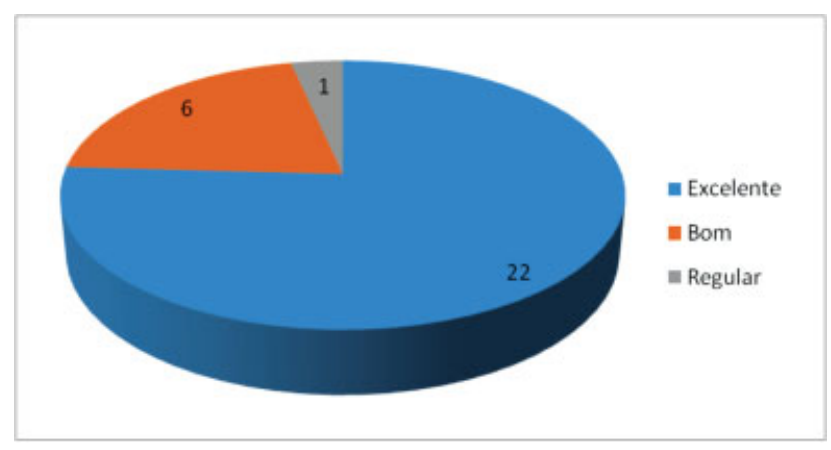

Fig. 1 Resultados das avaliações radiológicas.

obteve média de 59,43, DP de 9,86 e coeficiente de variação de $16,59 \%$. Já a extensão obteve média de 53,83, DP de 5,09 e coeficiente de variação de 9,46\% (-Tabela 3).

Não houve nenhum relato, nos prontuários dos pacientes, de complicações no pós-operatório.

\section{Discussão}

A fratura da extremidade distal do rádio é uma lesão complexa. Ela é mais frequente nos pacientes adultos após a $4^{\mathrm{a}}$ década de vida, perfazendo $\sim 10$ a $20 \%$ de todas as fraturas atendidas na emergência. A faixa etária mais acometida está entre os 60 e 69 anos, sendo as mulheres as principais vítimas. No entanto, percebe-se que, nos últimos 20 anos, um segundo pico de incidência surgiu entre aqueles com
Tabela 3 Média, desvio padrão e coeficiente de variação dos desvios ulnar e radial, flexão e extensão

\begin{tabular}{|l|l|l|l|l|}
\hline Estimativas & $\begin{array}{l}\text { Desvio } \\
\text { ulnar }\end{array}$ & $\begin{array}{l}\text { Desvio } \\
\text { radial }\end{array}$ & Flexão & Extensão \\
\hline Média & $28,40 \%$ & $22,93 \%$ & $59,43 \%$ & $53,83 \%$ \\
\hline Desvio padrão & $3,00 \%$ & $2,20 \%$ & $9,86 \%$ & $5,09 \%$ \\
\hline $\begin{array}{l}\text { Coeficiente } \\
\text { de variação }\end{array}$ & $10,56 \%$ & $9,59 \%$ & $16,59 \%$ & $9,46 \%$ \\
\hline
\end{tabular}

idades entre 20 e 40 anos. ${ }^{11-14}$ Em nosso estudo, verificou-se que as idades dos pacientes variaram de 20 a 91 anos, com média de 56 anos, sendo mais frequente em pacientes após a $5^{\text {a }}$ década de vida, se aproximando com as médias encontradas pelos autores mencionados acima.

Não existem dados epidemiológicos brasileiros, mas no Reino Unido sua incidência é de 9 por 10 mil homens e de 37 por 10 mil mulheres por ano. ${ }^{15}$ No presente trabalho, foram avaliados 20 pacientes do sexo feminino, correspondendo a $66,6 \%$ da amostra, e 10 pacientes do sexo masculino, correspondendo a 33,3\%, evidenciando a maior incidência deste tipo de fratura no sexo feminino. Em relação ao lado acometido, $63,3 \%$ das fraturas ocorreram no lado esquerdo, e $36,3 \%$ no lado direito.

Tem sido observado que a placa volar reduz o risco de irritação dos tendões, o que é um dos maiores problemas da placa dorsal. E alguns autores têm relatado poucas complicações utilizando esta placa. Entre as complicações do pósoperatório de fratura de rádio distal encontradas na literatura estão a tenossinovite de tendão extensor ou de tendão flexor, distrofia simpático reflexa, síndrome do túnel do carpo, atraso de consolidação, soltura do implante, rupturas tendíneas (tendão flexor longo do polegar ou do tendão extensor longo do polegar), consolidação viciosa, lesões neurais (nervo mediano) e artrose radiocárpica e radioulnar. ${ }^{8,12,16-22}$ Não foram observadas complicações em nosso trabalho

Leung et $\mathrm{al}^{23}$ reportaram melhores resultados funcionais e radiográficos com a utilização da placa volar bloqueada independentemente do tempo de seguimento pós-operatório observado em relação à utilização de fixadores externos ou de fixação percutânea com fios de Kirschner. $\mathrm{O}$ nosso trabalho corrobora com o autor quanto à obtenção de resultados funcionais, porém discordamos quanto ao tempo de seguimento de pós-operatório, pois observamos melhores resultados quando avaliamos após 6 meses.

Fujii et al $^{24}$ constataram não haver relação entre o resultado funcional com o restabelecimento dos parâmetros radiográficos da extremidade distal do rádio conforme eram anteriormente à fratura, assim como no estudo de Jaremko et al, ${ }^{25}$ que avaliaram pacientes submetidos ao tratamento conservador para fratura da extremidade distal do rádio e observaram que não houve relação entre os resultados funcionais e a redução da fratura até níveis radiográficos "aceitáveis." Xavier et al ${ }^{18}$ relataram que os valores radiológicos não influenciam na amplitude de movimento; nenhuma diferença significativa foi observada, pois, enquanto determinados pacientes com uma pequena mobilização do punho mostraram radiografias 
classificadas como boas ou excelentes, outros pacientes com ampla mobilização do punho apresentaram radiografias classificadas como regulares ou ruins de acordo com van Eerten et al. ${ }^{26}$ Drobetz et al, ${ }^{20}$ em seu estudo, obtiveram resultados radiográficos de um total de 30 pacientes; 24 foram classificados como bons ou excelentes, sendo 7 deles classificados como excelentes e 17 deles como bons de acordo com Lidström, o que correspondeu a $80 \%$ dos pacientes do estudo; 4 pacientes foram classificados como regulares e 2 casos como ruins. Severo et al, ${ }^{27}$ em estudo de fratura de rádio distal tratado pela técnica de Ulson, obtiveram 64 resultados considerados como excelentes (43,2\%), 62 bons ( $41,9 \%), 18$ regulares $(12,2 \%)$ e 4 pobres $(2,7 \%)$. A amplitude de movimento documentada obteve a média de flexão do punho de $65^{\circ}$ (normal até $80^{\circ}$ ), e de extensão de $61^{\circ}$ (normal até $70^{\circ}$ ), radiográficos através de análise estatística. $\mathrm{Na}$ avaliação radiográfica do nosso trabalho, foram encontrados 22 (75,86\%) resultados excelentes, $6(20,69 \%)$ bons e $1(3,45 \%)$ regular. Analisando os dados, pode-se dizer que praticamente houve sucesso dos procedimentos cirúrgicos, sendo que a maioria (75,86\%) obteve resultados radiográficos excelentes. E quanto ao arco de movimento, obtivemos como média para desvio ulnar $28,40^{\circ}$; desvio radial $22,93^{\circ}$; flexão $59,43^{\circ}$ e extensão $53,83^{\circ}$, o que nos leva a discordar de Fujii et al $^{24}$ e de Jaremko et $a l^{25}$ que citam não haver relação entre o resultado funcional com o restabelecimento dos parâmetros radiográficos da extremidade distal do rádio conforme eram anteriormente à fratura. Porém, no nosso estudo radiográfico, o coeficiente de variação demonstrou um resultado de dispersão homogêneo, corroborando com Drobetz et al ${ }^{20}$ e Severo et al, ${ }^{27}$ que em seus estudos obtiveram parâmetros radiográficos excelentes no pósoperatório.

\section{Conclusão}

Após a análise dos resultados, concluímos que:

- As fraturas de rádio distal acometem os pacientes na $5^{\underline{a}}$ década de vida.

- O sexo feminino foi o mais acometido.

- Houve um predomínio de acometimento do lado esquerdo.

- Após avaliação clínica e radiológica, a utilização de placa bloqueada é um método de tratamento com alto índice de sucesso no procedimento cirúrgico das fraturas de rádio distal.

Conflitos de Interesses

Os autores declaram não haver conflitos de interesses.

\section{Referências}

1 Arora R, Gabl M, Gschwentner M, Deml C, Krappinger D, Lutz M. A comparative study of clinical and radiologic outcomes of unstable colles type distal radius fractures in patients older than 70 years: nonoperative treatment versus volar locking plating. J Orthop Trauma 2009;23(04):237-242

2 O'Neill TW, Cooper C, Finn JD, Lunt M, Purdie D, Reid DM, et al; UK Colles' Fracture Study Group. Incidence of distal forearm fracture in British men and women. Osteoporos Int 2001;12(07):555-558
3 Liporace FA, Adams MR, Capo JT, Koval KJ. Distal radius fractures. J Orthop Trauma 2009;23(10):739-748

4 Osada D, Viegas SF, Shah MA, Morris RP, Patterson RM. Comparison of different distal radius dorsal and volar fracture fixation plates: a biomechanical study. J Hand Surg Am 2003;28(01): 94-104

5 McCall TA, Conrad B, Badman B, Wright T. Volar versus dorsal fixed-angle fixation of dorsally unstable extra-articular distal radius fractures: a biomechanic study. J Hand Surg Am 2007;32 (06):806-812

6 Wong KK, Chan KW, Kwok TK, Mak KH. Volar fixation of dorsally displaced distal radial fracture using locking compression plate. J Orthop Surg (Hong Kong) 2005;13(02):153-157

7 Jupiter JB, Marent-Huber M; LCP Study Group. Operative management of distal radial fractures with 2.4-millimeter locking plates. A multicenter prospective case series. J Bone Joint Surg Am 2009; 91(01):55-65

8 Arora R, Lutz M, Hennerbichler A, Krappinger D, Espen D, Gabl M. Complications following internal fixation of unstable distal radius fracture with a palmar locking-plate. J Orthop Trauma 2007;21 (05):316-322

9 Barton T, Chambers C, Bannister G. A comparison between subjective outcome score and moderate radial shortening following a fractured distal radius in patients of mean age 69 years. J Hand Surg Eur Vol 2007;32(02):165-169

10 Anzarut A, Johnson JA, Rowe BH, Lambert RG, Blitz S, Majumdar SR. Radiologic and patient-reported functional outcomes in an elderly cohort with conservatively treated distal radius fractures. J Hand Surg Am 2004;29(06):1121-1127

11 Caporrino FA, Belotti JC, Ulson HJR, Toledo LFQ Reis FB, Machado JKS. Fraturas da extremidade distal do rádio e da ulna. In: Pardini Júnior AG, Freitas A. Traumatismos da mão. 4a. ed. Rio de Janeiro: MedBook; 2008:411-445

12 de Oliveira Filho OM, Belangero WD, Teles JB. Fraturas do rádio distal: avaliação das classificações. Rev Assoc Med Bras (1992) 2004;50(01):55-61

13 Figl M, Weninger P, Liska M, Hofbauer M, Leixnering M. Volar fixed-angle plate osteosynthesis of unstable distal radius fractures: 12 months results. Arch Orthop Trauma Surg 2009;129 (05):661-669

14 Trumble TE, Wagner W, Hanel DP, Vedder NB, Gilbert M. Intrafocal (Kapandji) pinning of distal radius fractures with and without external fixation. J Hand Surg Am 1998;23(03):381-394

15 Nazar MA, Mansingh R, Bassi RS, Waseem M. Is there a consensus in the management of distal radial fractures? Open Orthop J 2009; 3:96-99

16 Orbay JL, Fernandez DL. Volar fixation for dorsally displaced fractures of the distal radius: a preliminary report. J Hand Surg Am 2002;27(02):205-215

17 Reis FB, Machado JKS. Fraturas da extremidade distal do rádio e da ulna - tratamento cruento. In: Pardini A, Freitas A. Traumatismos da Mão. Rio de Janeiro: Medbook; 2008:438-445

18 Xavier CRM, Molin DCD, Santos RMM, Santos RDT, Ferreira Neto JC. Tratamento cirúrgico das fraturas do rádio distal com placa volar bloqueada: correlação dos resultados clínicos e radiográficos. Rev Bras Ortop 2011;46(05):505-513

19 Kamano M, Koshimune M, Toyama M, Kazuki K. Palmar plating system for Colles' fractures-a preliminary report. J Hand Surg Am 2005;30(04):750-755

20 Drobetz H, Kutscha-Lissberg E. Osteosynthesis of distal radial fractures with a volar locking screw plate system. Int Orthop 2003;27(01):1-6

21 Rozental TD, Blazar PE. Functional outcome and complications after volar plating for dorsally displaced, unstable fractures of the distal radius. J Hand Surg Am 2006;31(03):359-365

22 Gruber G, Zacherl M, Giessauf C, Glehr M, Fuerst F, Liebmann W, et al. Quality of life after volar plate fixation of articular fractures 
308 Avaliação de pacientes submetidos a osteossíntese de rádio distal Câmara Filho, Ameida

of the distal part of the radius. J Bone Joint Surg Am 2010;92(05): $1170-1178$

23 Leung F, Zhu L, Ho H, Lu WW, Chow SP. Palmar plate fixation of AO type C2 fracture of distal radius using a locking compression plate-a biomechanical study in a cadaveric model. J Hand Surg [Br] 2003;28(03):263-266

24 Fujii K, Henmi T, Kanematsu Y, MishiroT, Sakai T, Terai T. Fractures of the distal end of radius in elderly patients: a comparative study of anatomical and functional results. J Orthop Surg (Hong Kong) 2002; 10(01):9-15
25 Jaremko JL, Lambert RG, Rowe BH, Johnson JA, Majumdar SR. Do radiographic indices of distal radius fracture reduction predict outcomes in older adults receiving conservative treatment? Clin Radiol 2007;62(01):65-72

26 van Eerten PV, Lindeboom R, Oosterkamp AE, Goslings JC. An X-ray template assessment for distal radial fractures. Arch Orthop Trauma Surg 2008;128(02):217-221

27 Severo LA, Mentz L, Busetto AM, Lech O, Pinto S. Fraturas da extremidade distal do rádio tratadas pela técnica de Ulson. Rev Bras Ortop 2003;38(08):438-445 